\title{
ORIGINAL
}

\section{APTITUD FÍSICA CARDIORRESPIRATORIA Y RIESGO CARDIOMETABÓLICO EN PERSONAS ADULTAS JÓVENES (*)}

Jeremías D Secchi (1) y Gastón C García (2).

(1) Profesorado de Educación Física. Universidad Adventista del Plata. Entre Ríos. Argentina.

(2) Instituto Superior de Formación Docente. San Rafael. Mendoza. Argentina.

(*) Invetigación financiada por la Universidad Adventista del Plata.

Los autores declaran que no existen conflictos de interés.

\section{RESUMEN}

Fundamentos: La evaluación del $\mathrm{VO}_{2 \max }$ permite clasificar a los sujetos según el riesgo para la salud. Sin embargo los factores que pueden afectar a las clasificaciones han sido poco estudiados. El objetivo de esta investigación fue determinar si el tipo de ecuación predictiva del $\mathrm{VO}_{2 \max }$ y los criterios de referencia del Fitnessgram ${ }^{\circledR}$ modifican la proporción de adultos jóvenes clasificados con un nivel de capacidad aeróbica indicativo de riesgo cardiometabólico.

Métodos: El diseño del estudio fue observacional, relacional y de corte transversal. Participaron voluntariamente 240 adultos jóvenes. El $\mathrm{VO}_{2}$ fue estimado mediante el test de Course Navette aplicando 9 ecuaciones predictivas. Las diferencias en las clasificaciones fueron analizadas con la prueba Q de Cochran y la de MacNemar.

Resultados: El nivel de capacidad aeróbica indicativo de riesgo cardiometabólico osciló entre $7,1 \%$ y 70,4\% según el tipo de ecuación predictiva y el criterio de referencia utilizado $(\mathrm{p}<0,001)$. Independientemente del la ecuación predictiva del $\mathrm{VO}_{2 \max }$ y el criterio de referencia aplicado; una mayor proporción de mujeres, del $29,4 \%$ al $85,3 \%$, fueron clasificadas con un nivel no saludable de capacidad aeróbica frente al $4,8 \%$ a $51 \%$ en varones $(\mathrm{p}<0,001)$. En ambos sexos, los viejos criterios de referencia clasificaron a una menor proporción de hombres (del $4,8 \%$ al $48,1 \%$ ) y mujeres (del $39,4 \%$ al $68,4 \%$ ) con capacidad aeróbica no saludable, independientemente de la ecuación aplicada $(\mathrm{p}<0,001)$.

Conclusiones: El tipo de ecuación predictiva del $\mathrm{VO}_{2}$ y los criterios de referencia del Fitnessgram ${ }^{*}$ modifican las clasificaciones de adultos jóvenes con un nivel de capacidad aeróbica saludable o de riesgo cardiometabólico.

Palabras clave: Consumo de oxígeno. Criterios de referencia. Síndrome X metabólico.

\section{ABSTRACT \\ Cardiorespiratory Fitness and Cardiometabolic Risk in Young Adults}

Background: The assessment of $\mathrm{VO}_{2 \max }$ allow classify subjects according to the health risk. However the factors that may affect the classifications have been little studied. The main purpose was to determine whether the type of $\mathrm{VO}_{2 \max }$ prediction equation and the Fitnessgram ${ }^{\mathbb{B}}$ criterion-referenced standards modified the proportion of young adults classified with a level of aerobic capacity cardiometabolic risk indicative.

Methods: The study design was observational, cross-sectional and relational. Young adults $(n=240)$ participated voluntarily. The $\mathrm{VO}_{2}$ was estimated by $20-\mathrm{m}$ shuttle run test applying 9 predictive equations. The differences in the classifications were analyzed with the Cochran Q and McNemar tests.

Results: The level of aerobic capacity indicative of cardiometabolic risk ranged between $7.1 \%$ and $70.4 \%$ depending on the criterion-referenced standards and predictive equation used $(\mathrm{p}<0.001)$. A higher percentage of women were classified with an unhealthy level in all equations (women: $29.4 \%$ to $85.3 \%$ vs $4.8 \%$ to $51 \%$ in men), regardless of the criterion-referenced standards $(\mathrm{p}<0.001)$. Both sexes and irrespective of the equation applied the old criterion-referenced standards classified a lower proportion of subjects (men: $4.8 \%$ to $48.1 \%$ and women: $39.4 \%$ a $68.4 \%$ ) with unhealthy aerobic capacity ( $\mathrm{p} \leq 0.004)$.

Conclusions: The type of $\mathrm{VO} 2$ max prediction equation and Fitnessgram $^{\circledR}$ criterion-referenced standards changed classifications young adults with a level of aerobic capacity of cardiometabolic risk indicative.

Keyword: Oxygen Consumption.Referenced standards. Metabolic syndrome X. Physical fitness. 


\section{INTRODUCCIÓN}

La aptitud física cardiorrespiratoria (AFC) es uno de los componentes más importantes de la condición física relacionada a la salud ${ }^{1,2}$. El $\mathrm{VO}_{2 \max }$ medido en un test incremental y máximo es considerado el "método de oro" para evaluar el componente cardiorrespiratorio ${ }^{3}$. Este ha sido motivo de estudio desde varios enfoques por estar relacionado con el rendimiento atlético ${ }^{4-6}$, la condición física y la salud ${ }^{7-11}$. La evaluación del $\mathrm{VO}_{2 \max }$ puede ser realizada con diversos propósitos, como determinar la capacidad funcional de un sujeto, monitorear la intensidad del ejercicio, verificar los efectos producidos por el entrenamiento y clasificar a las personas según el riesgo para la salud $^{11,12}$

Existe abundante evidencia científica que indica que la $\mathrm{AFC}$ es un importante indicador de salud en todas las edades ${ }^{7-10,13,14}$. Bajos niveles de $\mathrm{VO}_{2 \max }(<$ percentil 20) durante la edad adulta joven están relacionados con un riesgo 3 a 6 veces mayor para desarrollar hipertensión, síndrome metabólico (SM) y diabetes en la mediana edad en comparación con adultos jóvenes con altos niveles de $\mathrm{VO}_{2 \max }$ (>percentil 60) ${ }^{13}$. Estudios recientes indican que este marcador fisiológico no solo está relacionado a la morbilidad y mortalidad por enfermedad cardiovascular ${ }^{15}$, sino también con la calidad de vida ${ }^{16}$ y la salud mental ${ }^{17}$.

La fuerte relación establecida entre los niveles de AFC y varios resultados en la salud fundamentan la importancia de monitorear esta capacidad funcional en grandes poblaciones. Desafortunadamente la medición del $\mathrm{VO}_{2 \max }$, tanto en el laboratorio como en población general, hace necesario la utilización de analizadores de gases que limitan la evaluación por su elevado costo, duración y requerir técnicos calificados para llevarla a cabo ${ }^{18,19}$. Por esta razón, los tests de campo son una alternativa práctica y más viable para evaluar el componente car- diorrespiratorio en estudios epidemiológicos. En este sentido, el Course Navette (CN$20 \mathrm{~m}$ ) presenta algunas ventajas con respecto a otros tests, como la posibilidad de evaluar a gran cantidad de sujetos al mismo tiempo en un espacio reducido ${ }^{19}$, la validez y fiabilidad demostrada en un amplio rango de edades ( 8 a 47 años) ${ }^{19}$ y la estabilidad en la predicción del $\mathrm{VO}_{2 \max }$ en sujetos con distintos niveles de condición física ${ }^{20}$.

La manera de entender, evaluar e interpretar la condición física ha cambiado sustancialmente en los últimos 50 años ${ }^{21,22}$. Un área de reciente interés en el campo de la evaluación de la condición física esta enfocada a determinar la habilidad diagnóstica de los tests de campo o de laboratorio para clasificar correctamente a individuos con ciertos factores de riesgo o problemas de salud $^{2,22-25}$. Algunas de las razones que han llevado a este nuevo enfoque fueron las siguientes: 1) El cambio de paradigma de la aptitud física relacionada con el rendimiento a la aptitud física centrada en la salud ${ }^{22}$. 2) La transición de la evaluación basada en normas de referencia (NR) a la evaluación basada en criterios de referencia $(\mathrm{CR})^{22,26}$. 3) Por último, la incorporación de nuevas herramientas estadísticas en el campo de la evaluación de la condición física que han permitido estudiar las propiedades diagnósticas de los test de campo y validar los $\mathrm{CR}^{22}$.

Si bien la evaluación basada en CR fue introducida en el campo de la educación física a finales de la década de 1980, fue muy criticada porque en un principio se estableció en base a la opinión y el juicio de expertos sin que los CR tuvieran un sólido sustento o validación científica ${ }^{2,22-26}$. Recientemente, el Fitnessgram ${ }^{\circledR}$ ha validado los nuevos CR para la capacidad aeróbica en niños y adolescentes tomando como parámetro de salud el $\mathrm{SM}^{2}$. Estos nuevos $\mathrm{CR}$ se basan en el riesgo que un sujeto tiene para desarrollar SM si se encuentra por debajo o por encima de un determinado nivel de $\mathrm{VO}_{2 \max }{ }^{2}$. Hasta la actualidad no hay 
trabajos de investigación que hayan validado CR para la capacidad aeróbica en personas adultas jóvenes, adultas o adultas mayores.

Por otro lado, los factores que pueden afectar a las clasificaciones en niños y adolescentes a partir de la evaluación de la condición física utilizando tests de campo han sido poco estudiados ${ }^{22,24}$. Además esta línea de investigación aún no ha sido explorada en personas adultas jóvenes. Según nuestro conocimiento no existe ningún estudio publicado que haya analizado como puede afectar el tipo de ecuación predictiva del $\mathrm{VO}_{2 \max }$ y los CR del Fitnessgram ${ }^{\circledR}$ sobre las clasificaciones en cuanto al riesgo de salud en adultos jóvenes.

El objetivo principal del estudio fue determinar si las ecuaciones de Leger ${ }^{27}$, Stickland $^{28}$, Matsuzaka ${ }^{29}$, Flouris $^{30}$, Ruiz $^{31}$ y Chatterjee ${ }^{32,33}$ para predecir el $\mathrm{VO}_{2 \max }$ modifican la proporción de personas adultas jóvenes que son clasificadas con un nivel de $\mathrm{VO}_{2 \max }$ indicativo de riesgo cardiometabólico (RCM) según los CR (nuevos y viejos) propuestos por el Fitnessgram ${ }^{\circledR 2,26}$. E1 segundo objetivo fue conocer si había diferencias por sexo en las clasificaciones.

\section{SUJETOS Y MÉTODOS}

Diseño y muestra del estudio. Los sujetos fueron evaluados entre el 12 de octubre y el 23 de noviembre de 2010. El diseño fue observacional de corte transversal. La población de estudio estuvo conformada por 562 estudiantes de primer y segundo año de la Facultad de Ciencias de la Salud (FCS) de la Universidad Adventista del Plata (UAP), ubicada en la provinvia Entre Ríos, Argentina. De esta población, 213 $(37,9 \%)$ sujetos no desearon participar del estudio y $16(2,8 \%)$ fueron excluidos por presentar algún signo o síntoma de enfermedad cardiovascular (ECV) o problema musculo esquelético. Fueron excluidos 64 que no participaron el segundo día de evalua- ción y 29 que durante la prueba del CN-20m no realizaron un esfuerzo máximo, finalizando el test antes de alcanzar la fatiga debido a dolor muscular o articular, nauseas, mareos y falta de motivación. De esta manera, la muestra del estudio fue conformada por 240 estudiantes. Antes de aceptar la participación, fueron informados de forma verbal y por escrito acerca de los procedimientos, las evaluaciones, los beneficios y riesgos de participar en la investigación. El estudio contó con la aprobación de la comisión de bioética de la FCS de la UAP.

Procedimientos. La evaluación se realizó durante dos días alternos. Todos los tests fueron realizados por los mismos investigadores. El primer día se realizó el cuestionario de salud pre-actividad física (PAR-Q) y el cuestionario internacional de actividad física (IPAQ) versión corta ${ }^{34}$. Posteriormente, en el laboratorio, se tomó la frecuencia cardíaca (FC) y la presión arterial (PA) para luego realizar la evaluación antropométrica. El segundo día, en un gimnasio cubierto, se evaluó el CN-20m. Dos días previos a la evaluación, los participantes fueron informados de que debían abstenerse de realizar actividad física intensa, fumar, beber café o tomar alguna medicación. Los sujetos debían desayunar por lo menos dos horas antes de la administración de los tests y asistir con calzado y ropa deportiva. Todos los tests fueron administrados entre las 9:30 y las 11:30 am.

El nivel de actividad física se evaluó mediante el cuestionario IPAQ versión cor$\mathrm{ta}^{34}$. Los sujetos contestaron sobre la frecuencia y duración de la actividad física de intensidad vigorosa, moderada y la caminata durante la última semana. Se categorizó el nivel de actividad física en alto, moderado y bajo de acuerdo a las recomendaciones para este instrumento ${ }^{35}$.

Para la medición de la PA y la FC en reposo, los sujetos permanecieron sentados y sin hablar durante 5 minutos. La PA sistólica y 
diastólica fueron medidas 2 veces consecutivas usando un esfigmomanómetro de mercurio. Una tercera medición fue realizada si la diferencia entre las mediciones fue superior a $5 \mathrm{mmHg}$. La FC fue medida 2 veces con la técnica de palpación de la arteria radial durante 1 minuto. En el análisis se utilizó el promedio de las mediciones. Se emplearon todos los procedimientos para una correcta medición de la PA y la FC en reposo ${ }^{36}$.

Para la evaluación antropométrica los sujetos fueron pesados con la menor cantidad de ropa posible utilizando una balanza de pie marca CAM modelo P-1003, con resolución de $0,100 \mathrm{~kg}$. La estatura de parado fue medida con un estadiómetro siguiendo el protocolo ISAK $^{37}$. El Índice de Masa Corporal (IMC $\mathrm{kg} / \mathrm{m}^{2}$ ) fue calculado dividiendo el peso corporal del sujeto por su estatura expresada en metros al cuadrado. La medición del perímetro de cintura mínimo se realizó a nivel del punto mas estrecho entre el último arco costal y la cresta ilíaca al final de una espiración normal, con los brazos relajados a los costados del cuerpo ${ }^{37}$. Esta medición se efectuó con una cinta antropométrica de acero inextensible (W606PM, Lufkin, US).

La aptitud física cardiorrespiratoria fue evaluada mediante el test de Course Navette, también conocido como 20-m shuttle run test (20mSRT). Este, consiste en correr el mayor tiempo posible entre dos líneas separadas por $20 \mathrm{~m}$ en doble sentido, ida y vuelta. El ritmo de carrera es impuesto por una señal sonora. La velocidad inicial es de 8,5 $\mathrm{km} / \mathrm{h}^{-1}$ y se incrementa en $0,5 \mathrm{~km} / \mathrm{h}^{-1}$ a intervalos de 1 minuto. El sujeto debe pisar detrás de la línea de $20 \mathrm{~m}$ en el momento justo que se emite la señal sonora o "beep". El test finaliza cuando el sujeto se detiene porque alcanzó la fatiga o cuando por dos veces consecutivas no llega a pisar detrás de la línea al sonido del "beep". Los sujetos fueron alentados verbalmente para realizar el máximo esfuerzo. La relación evaluador- sujeto siempre fue $1: 1$. El $\mathrm{VO}_{2 \max }$ fue estimado a partir de 9 ecuaciones predictivas presentadas en el anexo 1. Los estudiantes fueron clasificados con un nivel de capacidad aeróbica saludable o indicativo de RCM de acuerdo a los viejos y nuevos CR establecidos por el Fitnessgram ${ }^{2,26}$ para la edad de 17 años o más:

- Viejos $\mathrm{CR}^{26}: \mathrm{VO}_{2 \max }$ de $42 \mathrm{ml} / \mathrm{kg}^{-1} / \mathrm{min}^{-1}$ para hombres y de $35 \mathrm{ml} / \mathrm{kg}-1 / \mathrm{min}^{-1}$ para mujeres.

- Nuevos CR 2 : $\mathrm{VO}_{2 \max }$ de $44,3 \mathrm{ml} / \mathrm{kg}^{-1} / \mathrm{min}^{-1}$ para hombres y de $38,6 \mathrm{ml} / \mathrm{kg}^{-1} / \mathrm{min}^{-1}$ para mujeres.

Análisis Estadístico. Los datos fueron analizados con el programa (SPSS) 18.0. La normalidad de las variables fue determinada con la prueba de Kolmogorov-Smirnov. Las diferencias entre sexos fueron determinadas con la prueba $\mathrm{T}$ de Students. Para determinar diferencias en el $\mathrm{VO}_{2 \max }$ predictivo entre las diferentes ecuaciones se utilizó la prueba $\mathrm{T}$ para muestras relacionadas.

Las diferencias en las clasificaciones entre las distintas ecuaciones predictivas del $\mathrm{VO}_{2 \max }$ y los CR del Fitnessgram ${ }^{\circledR}$ (viejos vs nuevos) fueron analizadas con la prueba $\mathrm{Q}$ de Cochran y la de MacNemar. La prueba de MacNemar fue aplicada para establecer entre 7 y 21 pares de comparaciones para determinar diferencias en las clasificaciones entre los nuevos vs los viejos CR y entre las distintas ecuaciones respectivamente. El nivel de significancia aceptado resultó de dividir el valor de $\mathrm{p} \leq 0,05$ por el número de pares de comparaciones realizado. Por ejemplo $(0,05 / 7=p \leq 0,007)$ para establecer diferencias entre proporciones comparando los nuevos y los viejos CR para cada ecuación. Para establecer diferencias en las clasificaciones entre las distintas ecuaciones se aceptó un nivel de significancia de $(0,05 / 21=p \leq 0,002)$. Las diferencias entre sexos en las categorías fueron determinadas aplicando la prueba U de Mann-Whitney. 
En este caso y para el análisis de comparación de medias el nivel de significancia aceptado fue de $\mathrm{p}<0,05$.

\section{RESULTADOS}

De la muestra de estudio, $136(56,7 \%)$ fueron mujeres. Las características de los sujetos se presentan en la tabla $1 . \mathrm{El} \mathrm{VO}_{2 \max }$ predictivo fue mayor en los hombres en todas las ecuaciones (tabla 1). Las diferencias promedio fueron de $8,9-12,2 \mathrm{ml} / \mathrm{kg}^{-}$ ${ }^{1 /} \mathrm{min}^{-1}(18,3 \%-24,8 \%)$.

$\mathrm{El} \mathrm{VO}_{2 \max }$ predictivo fue diferente entre las distintas ecuaciones $(\mathrm{p}<0,001)$, con excepción de Stickland vs Matsuzaka (a) $(p=0,202)$. En los varones no se apreciaron diferencias en el $\mathrm{VO}_{2 \max }$ predictivo entre Stickland vs Matsuzaka (a) $(\mathrm{p}=0,666)$. Stickland vs Ruiz ( $\mathrm{p}=0,073$ ) y Matsuzsaka (a) vs Ruiz $(p=0,140)$. En las mujeres no hubo diferencias entre Stickland vs Matsuzaka (b) $(\mathrm{p}=0,320)$ y Matsuzaka (a) vs Matsuzaka (b) $(\mathrm{p}=0,078)$.

La figura 1 muestra los niveles de actividad física en total y por sexo. La prueba $U$ de Mann - Whitney mostró diferencias estadísticamente significativas en la prevalencia de los niveles de actividad física entre $\operatorname{sexos}(\mathrm{p}<0,05)$. Para el nivel bajo fue $38,1 \%$ vs $47,8 \%$ y para el nivel alto fue $24 \%$ vs $12,5 \%$ para hombres y mujeres respectivamente.

La figura 2 muestra que la proporción de estudiantes con RCM osciló entre el 30,4\% y 70,4\% según los nuevos CR y entre el $7,1 \%$ al $59,4 \%$ para los viejos CR. La prueba $\mathrm{Q}$ de Cochran mostró diferencias significativas entre las proporciones para los nuevos $(p<0,001)$ y para los viejos $C R$ del Fitnessgram $^{\circledR}$ (figura 2).

En la tabla 2 se pueden apreciar las diferencias en las clasificaciones según la prueba de MacNemar. La ecuación de Leger clasificó al 70,4\% de los sujetos para los nuevos CR y 59,4\% para los viejos CR con capacidad aeróbica no saludable. Las diferencias fueron estadísticamente significativas con respecto a las demás ecuaciones utilizadas $(\mathrm{p}<0,001)$ con excepción de la de Flouris (tabla 2). Por otro lado, la ecuación de Ruiz clasificó al $30,4 \%$ de los sujetos para los nuevos CR y al $7,1 \%$ según los viejos $\mathrm{CR}$ con nivel de capacidad aeróbica no saludable. Se observaron diferencias estadísticamente significativas entre la ecuación de Ruiz y las otras ecuaciones aplicadas $(p<0,001)$. No se observaron diferencias estadísticamente significativas entre las siguientes ecuaciones: Stickland vs Matsuzaka (a), Stickland vs Matsuzaka (b); Matsuzaka (a) vs Matsuzaka (b) cuando se utilizaron ambos CR $(\mathrm{p}>0,002)$.

Como se muestra en la tabla 2, independientemente del tipo de ecuación aplicada del $7,1 \%$ al $59,4 \%$ de los sujetos fueron clasificados con un nivel de capacidad aeróbica indicativo de RCM cuando se emplearon los viejos CR $(\mathrm{p}<0,001)$.

La tabla 3 muestra las proporciones de sujetos con capacidad aeróbica saludable y no saludable de acuerdo al sexo, la ecuación aplicada y los CR del Fitnessgram ${ }^{\circledR}$. Entre el $29,4 \%$ al $85,3 \%$ de las mujeres vs $4,8 \%$ a $51 \%$ en hombres fueron clasificados con un nivel de capacidad aeróbica no saludable en todas las ecuaciones, independientemente del CR empleado. La prueba de U Mann- Whitney mostró diferencias estadísticamente significativas entre sexos para todas las ecuaciones en ambos CR $(\mathrm{p}<0,001)$.

En hombres y mujeres por separado la prueba de MacNemar mostró significativas en las proporciones de sujetos con niveles saludables y no saludables entre los nuevos vs los viejos CR en todas las ecuaciones $(p \leq 0,004)$ con excepción de la ecuación de Leger en hombres $(\mathrm{p}>0,007)$ (tabla 3). 
Tabla 1

Características de los sujetos

\begin{tabular}{|c|c|c|c|c|c|c|c|}
\hline \multirow[b]{2}{*}{ Características generales } & \multicolumn{2}{|c|}{ Todos $(n=240)$} & \multicolumn{2}{|c|}{ Hombres $(n=104)$} & \multicolumn{2}{|c|}{ Mujeres $(n=136)$} & \multirow{2}{*}{$\mathrm{P}$} \\
\hline & Media & DS & Media & DS & Media & DS & \\
\hline Edad (años) & 19,9 & 2,3 & 20,1 & 2,4 & 19,6 & 2,2 & 0,098 \\
\hline Peso $(\mathrm{kg})$ & 65,2 & 11,3 & 71,1 & 9,4 & 60,7 & 10,6 & 0,001 \\
\hline Talla $(\mathrm{cm})$ & 167,8 & 9,1 & 173,9 & 6,8 & 163,1 & 7,8 & 0,001 \\
\hline PAS (mmHg) & 109,3 & 11,7 & 115,1 & 10,1 & 104,9 & 10,8 & 0,001 \\
\hline $\mathrm{PAD}(\mathrm{mmHg})$ & 67,5 & 9,4 & 70,5 & 9,1 & 65,2 & 8,9 & 0,001 \\
\hline FC reposo (bpm) & 72,6 & 10,4 & 70,8 & 9,1 & 73,9 & 11,1 & 0,022 \\
\hline \multicolumn{8}{|l|}{ Indicadores de obesidad } \\
\hline $\mathrm{IMC}\left(\mathrm{Kg} / \mathrm{m}^{2}\right)$ & 23,1 & 3,1 & 23,5 & 2,9 & 22,8 & 3,2 & 0,069 \\
\hline Perímetro de cintura $(\mathrm{cm})$ & 74,8 & 8,6 & 78,6 & 8 & 71,8 & 8 & 0,001 \\
\hline \multicolumn{8}{|l|}{ Course Navette } \\
\hline Distancia (m) & 980,2 & 450,7 & 1341 & 383,4 & 704,4 & 265,7 & 0,001 \\
\hline $\mathrm{N}^{\circ}$ de pasadas & 49 & 22,5 & 67 & 19,2 & 35,2 & 13,3 & 0,001 \\
\hline Etapa (nivel) & 6,4 & 2,4 & 8,3 & 1,9 & 5 & 1,5 & 0,001 \\
\hline Velocidad máx. $\left(\mathrm{km} / \mathrm{h}^{-1}\right)$ & 11,2 & 1,2 & 12,1 & 1,0 & 10,5 & 0,8 & 0,001 \\
\hline Velocidad $\left(\mathrm{km} / \mathrm{h}^{-1}\right)^{*}$ & 10,8 & 1,2 & 11,7 & 1,0 & 10 & 0,7 & 0,001 \\
\hline \multicolumn{8}{|l|}{$\mathrm{VO}_{2 \max }\left(\mathrm{ml} / \mathrm{kg}^{-1} \mathrm{~min}^{-1}\right)$} \\
\hline Leger y Gadoury, 1989 & 37,2 & 7,1 & 42,8 & 5,8 & 32,8 & 4,5 & 0,001 \\
\hline Stickland et al., 2003 & 42,3 & 7,6 & 49,2 & 5,1 & 37 & 4,3 & 0,001 \\
\hline Matsuzaka (a) et al., 2004 & 42,4 & 7,6 & 49,1 & 5,4 & 37,3 & 4,5 & 0,001 \\
\hline Matsuzaka (b) et al., 2004 & 41,6 & 6,9 & 47,5 & 5,2 & 37,1 & 4,1 & 0,001 \\
\hline Flouris et al., 2005 & $\begin{array}{ll}---- \\
\end{array}$ & 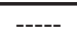 & 44 & 6,4 & $\begin{array}{ll}---- \\
\end{array}$ & $\begin{array}{ll}---- \\
\end{array}$ & $\begin{array}{ll}---- \\
\end{array}$ \\
\hline Ruiz et al., 2008 & 43,6 & 6 & 48,7 & 4,5 & 39,8 & 3,7 & 0,001 \\
\hline Chatterjee et al., 2010 & 39,5 & 6,6 & 45,3 & 5 & 35,1 & 3,6 & 0,001 \\
\hline \multicolumn{8}{|l|}{ Tipo de actividad física } \\
\hline Vigorosa $(d \cdot s e m)$ & 1,8 & 1,9 & 2,1 & 2 & 1,5 & 1,7 & 0,026 \\
\hline Vigorosa(d·sem) & 99,5 & 134,1 & 130,4 & 164,5 & 75,8 & 99,4 & 0,002 \\
\hline Moderada (min $\cdot$ sem $)$ & 1,7 & 1,9 & 1,9 & 2,0 & 1,5 & 1,9 & 0,153 \\
\hline Moderada (min·sem) & 66,7 & 89,8 & 77,1 & 100,4 & 58,8 & 80,3 & 0,117 \\
\hline Caminata (min·sem) & 2,8 & 2,4 & 2,7 & 2,5 & 2,8 & 2,4 & 0,789 \\
\hline Caminata (min·sem) & 81,2 & 99,1 & 76,3 & 98,8 & 84,9 & 99,5 & 0,511 \\
\hline Totales METs'hs'sem & 22,8 & 23,2 & 27,1 & 28,1 & 19,4 & 18,1 & 0,012 \\
\hline Tiempo sentado (hs·d) & 10,2 & 2,5 & 9,7 & 2,4 & 10,5 & 2,5 & 0,018 \\
\hline
\end{tabular}

$\mathrm{DS}=$ desviación estandar. $\mathrm{FC}$ reposo $=$ frecuencia cardíaca de reposo. $\mathrm{IMC}=$ índice de masa corporal. $\mathrm{N}^{\circ}$ de pasadas $=$ corresponde al número de vueltas de 20 metros en el Course Navette. PAD= presión arterial diastólica. PAS= presión arterial sistólica. *Velocidad en $\mathrm{km} \cdot \mathrm{h}^{-1}$ considerando la última etapa completa en el test de Course $\mathrm{Navette} \mathrm{VO}_{2 \max }=$ Consumo máximo de oxígeno predictivo. 
Figura 1

Niveles de actividad física según el cuestionario IPAQ versión corta

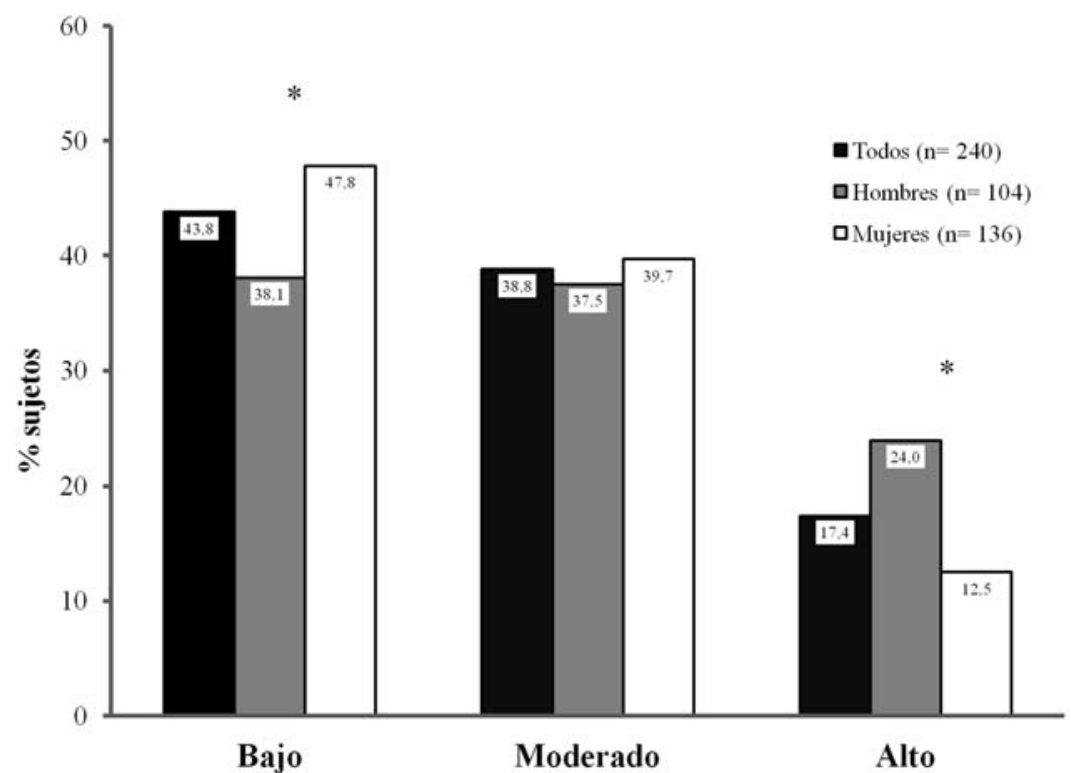

*Diferencias estadísticamente significativas en los niveles de actividad física entre hombres y mujeres $(\mathrm{p}<0,05)$.

\section{Figura 2}

Adultos jóvenes clasificados con un nivel de capacidad aeróbica indicativo de riesgo cardiometabólico de acuerdo a los nuevos y viejos criterios de referencia del Fitnessgram $^{\circledR}$ y las diferentes ecuaciones predictivas del $\mathrm{VO}_{2 \max }$ utilizadas

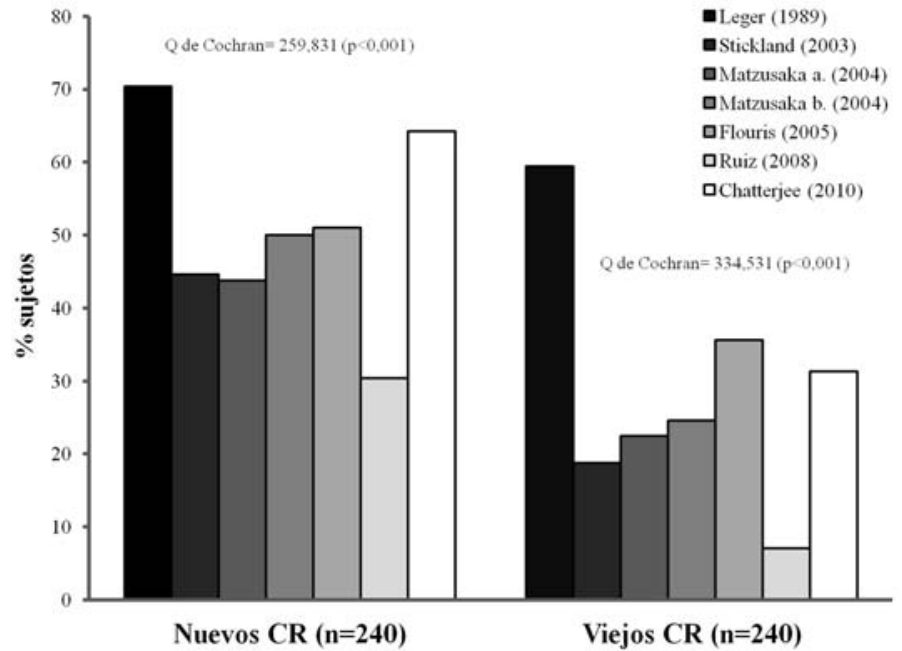

La prueba Q de Cochran muestra diferencias significativas entre las proporciones cuando se emplearon ambos criterios de referencia del Fitnessgram ${ }^{\circledR 2,26}$. La ecuación de Flouris solo fue aplicada en hombres $(\mathrm{n}=104)$. 
Tabla 2

Clasificación de los sujetos con un nivel de capacidad aeróbica saludable y no saludable según diferentes ecuaciones predictivas del VO2max y de acuerdo a los dos criterios de referencia propuestos por el Fitnessgram ${ }^{\circledR 2,26}$.

\begin{tabular}{|c|c|c|c|}
\hline $\begin{array}{l}\text { Ecuaciones predictivas } \\
\text { del } \mathrm{VO}_{2 \max }\end{array}$ & $\begin{array}{c}\text { Nuevos CR } \\
\text { Todos }(\mathrm{n}=240)\end{array}$ & $\begin{array}{c}\text { Viejos CR } \\
\text { Todos }(n=240)\end{array}$ & $\mathrm{P}^{*}$ \\
\hline $\begin{array}{l}\text { Leger et al, } 1989 \\
\text { No Saludable (\%) } \\
\text { Saludable (\%) }\end{array}$ & $\begin{array}{l}70,4^{\mathrm{a}} \\
29,6^{\mathrm{a}}\end{array}$ & $\begin{array}{l}59,6^{\mathrm{a}} \\
40,4^{\mathrm{a}}\end{array}$ & $\begin{array}{l}0,001 \\
0,001\end{array}$ \\
\hline $\begin{array}{l}\text { Stickland et a, } 2003 \\
\text { No Saludable (\%) } \\
\text { Saludable (\%) }\end{array}$ & $\begin{array}{l}44,6^{\mathrm{b}} \\
55,4^{\mathrm{b}}\end{array}$ & $\begin{array}{l}18,8^{\mathrm{b}} \\
81,2^{\mathrm{b}}\end{array}$ & $\begin{array}{l}0,001 \\
0,001\end{array}$ \\
\hline $\begin{array}{c}\text { Matsuzaka (a) et al, } 2004 \\
\text { No Saludable (\%) } \\
\text { Saludable (\%) }\end{array}$ & $\begin{array}{l}43,8^{\mathrm{c}} \\
56,2^{\mathrm{c}}\end{array}$ & $\begin{array}{l}22,5^{\mathrm{g}} \\
77,5^{\mathrm{g}}\end{array}$ & $\begin{array}{l}0,001 \\
0,001\end{array}$ \\
\hline $\begin{array}{c}\text { Matsuzaka (b) et al, } 2004 \\
\text { No Saludable (\%) } \\
\text { Saludable (\%) }\end{array}$ & $\begin{array}{l}50,0^{d} \\
50,0^{d}\end{array}$ & $\begin{array}{l}24,6^{\mathrm{h}} \\
75,4^{\mathrm{h}}\end{array}$ & $\begin{array}{l}0,001 \\
0,001\end{array}$ \\
\hline $\begin{array}{c}\text { Flouris et al., } 2005 \# \\
\text { No Saludable (\%) } \\
\text { Saludable (\%) }\end{array}$ & $\begin{array}{l}51,0^{\mathrm{e}} \\
49,0^{\mathrm{e}}\end{array}$ & $\begin{array}{l}35,6^{\mathrm{e}} \\
64,4^{\mathrm{e}}\end{array}$ & $\begin{array}{l}0,001 \\
0,001\end{array}$ \\
\hline $\begin{array}{c}\text { Ruiz et al, } 2008 \\
\text { No Saludable }(\%) \\
\text { Saludable }(\%)\end{array}$ & $\begin{array}{l}30,4^{\mathrm{f}} \\
69,6^{\mathrm{f}}\end{array}$ & $\begin{array}{l}7,1^{\mathrm{f}} \\
92,9^{\mathrm{f}}\end{array}$ & $\begin{array}{l}0,001 \\
0,001\end{array}$ \\
\hline $\begin{array}{c}\text { Chatterjee et al, } 2010 \\
\text { No Saludable (\%) } \\
\text { Saludable (\%) }\end{array}$ & $\begin{array}{l}64,2 \\
35,8\end{array}$ & $\begin{array}{l}31,2 \\
68,8\end{array}$ & $\begin{array}{l}0,001 \\
0,001\end{array}$ \\
\hline
\end{tabular}

Nuevos CR y Viejos $\mathrm{CR}=$ nuevos y viejos criterios de referencia para la capacidad aeróbica según el Fitness$\operatorname{gram}^{\circledR 2,26}$. \#La ecuación de Flouris fue aplicada solamente en hombres. * Valor de significancia estadística entre los Nuevos CR y los Viejos CR según la prueba de MacNemar. Para comprobar diferencias en las proporciones entre las diferentes ecuaciones se realizaron 21 pares de comparaciones aplicando la prueba de MacNemar: a. Leger diferente de Stickland; Matsuzaka (a); Matsuzaka (b), Ruiz y Chatterjee ( $\mathrm{p}<0,001)$. b. Stickland diferente de Flouris; Ruiz y Chatterjee $(\mathrm{p}<0,001)$. c. Matsuzaka (a) diferente de Matsuzaka (b); Flouris; Ruiz y Chatterjee $(p<0,001)$. d. Matsuzaka (b) diferente de Flouris; Ruiz y Chatterjee $(p<0,001)$. e. Flouris diferente de Ruiz y Chatterjeee $(p<0,001)$. f. Ruiz diferente de Chatterjee $(p<0,001)$. g. Matsuzaka (a) diferente de Flouris, Ruiz y Chatterjee $(\mathrm{p}<0,001)$. h. Matsuzaka (b) diferente de Flouris y Ruiz $(\mathrm{p}<0,001)$. 
Tabla 3

Clasificación de los sujetos con un nivel de capacidad aeróbica no saludable y saludable según diferentes ecuación predictivas del $\mathrm{VO}_{2 \max }$ y de acuerdo a los nuevos y viejos criterios de referencia propuestos por el Fitnessgram ${ }^{\circledR 2,26}$ en ambos sexos

\begin{tabular}{|c|c|c|c|c|c|c|}
\hline \multirow{2}{*}{$\begin{array}{l}\text { Ecuaciones predictivas } \\
\text { del } \mathrm{VO}_{2 \max }\end{array}$} & \multicolumn{3}{|c|}{ Nuevos CR } & \multicolumn{3}{|c|}{ Viejos CR } \\
\hline & $\begin{array}{c}\text { Hombres } \\
(n=104)\end{array}$ & $\begin{array}{l}\text { Mujeres } \\
(\mathrm{n}=136)\end{array}$ & $\mathrm{p}^{*}$ & $\begin{array}{c}\text { Hombres } \\
(\mathrm{n}=104)\end{array}$ & $\begin{array}{l}\text { Mujeres } \\
(\mathrm{n}=136)\end{array}$ & $\mathrm{p}^{*}$ \\
\hline $\begin{array}{c}\text { Leger et al, } 1989 \\
\text { No Saludable }(\%) \\
\text { Saludable }(\%)\end{array}$ & $\begin{array}{l}51,0^{\mathrm{a}} \\
49,0^{\mathrm{a}}\end{array}$ & $\begin{array}{l}85,3 \mathrm{~g} \dagger \\
14,7 \mathrm{~g} \dagger\end{array}$ & $\begin{array}{l}0,001 \\
0,001\end{array}$ & $\begin{array}{l}48,1^{\mathrm{a}} \\
51,9^{\mathrm{a}}\end{array}$ & $\begin{array}{l}68,4^{\mathrm{g}} \\
31,6^{\mathrm{g}}\end{array}$ & $\begin{array}{l}0,001 \\
0,001\end{array}$ \\
\hline $\begin{array}{c}\text { Stickland et al., } 2003 \\
\text { No Saludable }(\%) \\
\text { Saludable }(\%)\end{array}$ & $\begin{array}{l}15,4^{\mathrm{b} \dagger} \\
84,6^{\mathrm{b} \dagger}\end{array}$ & $\begin{array}{l}66,9 \mathrm{~h} \dagger \\
33,1 \mathrm{~h} \dagger\end{array}$ & $\begin{array}{l}0,001 \\
0,001\end{array}$ & $\begin{array}{c}4,8 \mathrm{~b} \\
95,2 \mathrm{~b}\end{array}$ & $\begin{array}{l}29,4^{\mathrm{h}} \\
70,6^{\mathrm{h}}\end{array}$ & $\begin{array}{l}0,001 \\
0,001\end{array}$ \\
\hline $\begin{array}{c}\text { Matsuzaka (a) et al., } 2004 \\
\text { No Saludable (\%) } \\
\text { Saludable (\%) }\end{array}$ & $\begin{array}{l}20,2^{\mathrm{c}} \\
78,8^{\mathrm{c}}\end{array}$ & $\begin{array}{l}61,8^{\mathrm{i} \uparrow} \\
38,2^{\mathrm{i} \dagger}\end{array}$ & $\begin{array}{l}0,001 \\
0,001\end{array}$ & $\begin{array}{l}11,5^{\mathrm{k}} \\
88,5^{\mathrm{k}}\end{array}$ & $\begin{array}{l}30,9^{\mathrm{i}} \\
69,1^{\mathrm{i}}\end{array}$ & $\begin{array}{l}0,001 \\
0,001\end{array}$ \\
\hline $\begin{array}{c}\text { Matsuzaka (b) et al., } 2004 \\
\text { No Saludable }(\%) \\
\text { Saludable }(\%)\end{array}$ & $\begin{array}{l}27,9^{\mathrm{d} \dagger} \\
72,1^{\mathrm{d} \dagger}\end{array}$ & $\begin{array}{l}66,9^{\mathrm{j} \dagger} \\
33,1^{\mathrm{j} \dagger}\end{array}$ & $\begin{array}{l}0,001 \\
0,001\end{array}$ & $\begin{array}{l}16,3^{1} \\
83,7^{1}\end{array}$ & $\begin{array}{l}30,9^{j} \\
69,1^{j}\end{array}$ & $\begin{array}{l}0,001 \\
0,001\end{array}$ \\
\hline $\begin{array}{c}\text { Flouris et al., } 2005 \# \\
\text { No Saludable }(\%) \\
\text { Saludable }(\%)\end{array}$ & $\begin{array}{l}51,0^{\mathrm{e}}{ }^{\dagger} \\
49,0^{\mathrm{e}}\end{array}$ & - & - & $\begin{array}{l}35,6^{\mathrm{e}} \\
64,6^{\mathrm{e}}\end{array}$ & - & $\begin{array}{l}- \\
-\end{array}$ \\
\hline $\begin{array}{l}\text { Ruiz et al., } 2008 \\
\text { No Saludable (\%) } \\
\text { Saludable (\%) }\end{array}$ & $\begin{array}{l}15,4^{\text {f }} \\
84,6^{\text {f }}\end{array}$ & $\begin{array}{l}66,9^{\mathrm{f}}{ }^{\dagger} \\
32,1^{\mathrm{f}}\end{array}$ & $\begin{array}{l}0,001 \\
0,001\end{array}$ & $\begin{array}{l}4,8^{\mathrm{f}} \\
95,2^{\mathrm{f}}\end{array}$ & $\begin{array}{l}29,4^{\mathrm{f}} \\
70,6^{\mathrm{f}}\end{array}$ & $\begin{array}{l}0,001 \\
0,001\end{array}$ \\
\hline $\begin{array}{c}\text { Chatterjee et al., } 2010 \\
\text { No Saludable }(\%) \\
\text { Saludable }(\%)\end{array}$ & $\begin{array}{l}37,5^{\dagger} \\
62,5^{\dagger}\end{array}$ & $\begin{array}{l}84,6^{\dagger} \\
15,4^{\dagger}\end{array}$ & $\begin{array}{l}0,001 \\
0,001\end{array}$ & $\begin{array}{l}17,3 \\
82,7\end{array}$ & $\begin{array}{l}41,9 \\
58,1\end{array}$ & $\begin{array}{l}0,001 \\
0,001\end{array}$ \\
\hline
\end{tabular}

La prueba U de Mann-Wihtney fue aplicada para establecer diferencias estadísticamente significativas en las clasificaciones entre ambos sexos. ${ }^{*}, * *$ Valor de significancia estadística entre sexos según los nuevos y viejos CR del Fitnessgram ${ }^{\mathbb{R}}$ respectivamente. $\dagger$ Diferencias significativas entre las proporciones según los Nuevos CR vs los Viejos CR para la prueba de MacNemar $p \leq 0,004$. Para comprobar diferencias en las proporciones entre las diferentes ecuaciones se realizaron entre 15 a 21 pares de comparaciones aplicando la prueba de MacNemar: a. Leger diferente de Stickland, Matsuzaka (a); Matsuzaka (b), Ruiz y Chatterjee ( $p<0,001)$. b. Stickland diferente de Matsuzka (b), Flouris y Chatterjee ( $\mathrm{p} \leq 0,002)$. c. Matsuzaka (a) diferente de Flouris y Chatterjee ( $<<0,001)$. d. Matsuzaka (b) diferente de Flouris y Chatterjee $(\mathrm{p} \leq 0,002)$. e. Flouris diferente de Ruiz y Chatterjee $(\mathrm{p}<0,001)$. f. Ruiz diferente de Chatterjee ( $\mathrm{p} \leq 0,001)$. g. Leger diferente de Stickland, Matsuzaka (a), Matsuzaka (b), y Ruiz ( $\mathrm{p}<0,001)$. h. Stickland diferente de Ruiz y Chatterjee $(p<0,001)$. i. Matsuzaka (a) diferente de Ruiz y Chatterjee $(p<0,001)$. j. Matsuzaka (b) diferente de Ruiz y Chatterjee ( $<<0,001)$. k. Matsuzaka (a) diferente de Flouris $(\mathrm{p}<0,001)$. 1. Matsuzaka (b) diferente de Flouris y Ruiz ( $\mathrm{p} \leq 0,002)$. 


\section{DISCUSIÓN}

El presente trabajo de investigación es el primero en demostrar que el tipo de ecuación predictiva del $\mathrm{VO}_{2 \max }$ y los CR del Fitnessgram $^{\circledR}$ modifican las clasificaciones de adultos jóvenes con nivel de capacidad aeróbica saludable o indicativo de RCM.

La ecuación predictiva del $\mathrm{VO}_{2 \max }$ aplicada es un factor que debe ser considerado a la hora de utilizar la evaluación de la AFC con fines diagnósticos. Por ejemplo, en el presente estudio las ecuaciones de $\operatorname{Leger}^{27}$ y Ruiz ${ }^{31}$ arrojaron resultados extremos. La variabilidad en las clasificaciones podría estar relacionada con las diferencias en la precisión y el error de predicción de las diferentes ecuaciones aplicadas. Varios estudios encontraron que la ecuación de Leger para adultos subestima el $\mathrm{VO}_{2 \max }$ medido en un rango de $-2,3$ a $-7,4 \mathrm{ml} / \mathrm{kg}^{-1} \cdot \mathrm{min}^{-}$ $120,28,38,39$. En el presente estudio la ecuación de Leger arrojó los valores de $\mathrm{VO}_{2 \text { max }}$ predictivo mas bajos en comparación con las demás ecuaciones aplicadas. Este hecho podría explicar por qué la ecuación de Leger clasificó a un mayor porcentaje de sujetos con niveles de capacidad aeróbica no saludable. Por otro lado, el $\mathrm{VO}_{2 \max }$ predictivo promedio obtenido a partir de la ecuación de Ruiz arrojó los valores más altos y por esta razón una menor proporción de sujetos fueron clasificados con un nivel de capacidad aeróbica de RCM. Es oportuno destacar que las variables incluidas para estimar el $\mathrm{VO}_{2 \max }$, el (EEE), las formas de considerar las etapas y la velocidad final alcanzada en el CN-20m varían entre las distintas ecuaciones aplicadas (anexo 1). Estas diferencias junto a las distintas metodologías aplicadas para confeccionar las ecuaciones podrían explicar, al menos en parte, la gran variabilidad producida en las clasificaciones.

Es importante mencionar que el presente estudio no evaluó la precisión de las diferentes ecuaciones para identificar SM o problemas cardiovasculares. Hasta el momento esta temática ha sido abordada solamente en adolescentes como lo muestra el trabajo de Morei- $\mathrm{ra}^{24}$. En su estudio se analizó la habilidad diagnóstica de 5 ecuaciones predictivas del $\mathrm{VO}_{2 \max }$ empleando análisis ROC para determinar bajo $o$ alto riesgo metabólico en adolescentes Portugueses ${ }^{24}$. Los resultados mostraron que las ecuaciones de Barnnett (a) y Matsuzaka tienen la mejor relación entre sensibilidad y especificidad para discriminar riesgo metabólico en adolescentes (en ambos sexos) y la ecuación de Ruiz resultó ser la mas válida para varones ${ }^{24}$.

Independientemente de la ecuación de predicción aplicada o el CR empleado, un mayor porcentaje de mujeres fueron clasificadas con capacidad aeróbica no saludable. Estos resultados podrían estar relacionados con las diferencias en los niveles de actividad física entre sexos, específicamente con la frecuencia y duración de la actividad física vigorosa que fue significativamente mayor en hombres. Varios estudios experimentales han mostrado que los mayores incrementos en el $\mathrm{VO}_{2 \max }$ se obtienen con programas de entrenamiento aeróbico de intensidades vigorosas y máximas $^{40-42}$.

En el presente estudio, las diferencias promedio en el $\mathrm{VO}_{2 \max }$ predictivo entre sexos oscilaron entre el $18,3 \%$ y $24,8 \%$ según la ecuación aplicada. En concordancia con estos resultados, Garcia y Secchi ${ }^{19}$ encontraron que el $\mathrm{VO}_{2 \max }$ predictivo fue un $23,5 \%$ inferior en las mujeres. Otras investigaciones han mostrado diferencias en la potencia aeróbica entre el $11 \%$ y el $23,8 \% 0^{13,20,28,29}$. Los menores niveles de $\mathrm{VO}_{2 \text { max }}$ observados en las mujeres pueden estar relacionados con el menor tamaño corporal, la masa muscular, el volumen sanguíneo, la concentración de hemoglobina, el volumen sistólico, el gasto cardíaco y un mayor porcentaje de grasa en comparación con los hombres $^{43}$.

Los resultados del presente estudio mostraron que independientemente de la ecuación aplicada (con excepción de la ecuación de Leger en hombres) un porcentaje significativamente menor de adultos jóvenes fue clasifi- 
cado con RCM cuando se emplearon los viejos CR. Estos resultados fueron de esperarse debido a que, para la edad de 17 años o más, los valores del $\mathrm{VO}_{2 \max }$ umbral de los viejos $\mathrm{CR}^{26}$ son menores a los nuevos $\mathrm{CR}^{2}$.

Recientemente Welk et al, compararon las diferencias en las clasificaciones empleando los CR del Fitnessgram ${ }^{\circledR}$, utilizando el test de 1 milla y el CN-20m en niños y adolescentes ${ }^{25}$. Los resultados mostraron que un alto porcentaje de los sujetos más jóvenes alcanzó la zona saludable en ambos CR. De todas maneras, una menor proporción de adolescentes mayores alcanzaron el nivel saludable según los CR del Fitnessgram ${ }^{\circledR 25}$. Además se observaron diferencias en las clasificaciones entre ambos tests. Según nuestro conocimiento no existe ninguna investigación publicada que haya analizado las diferencias en las clasificaciones al utilizar los viejos y nuevos CR del Fitnessgram $^{\circledR}$ en adultos jóvenes.

Cuando se utiliza CN-20m con fines diagnósticos nos enfrentamos con algunos problemas y preguntas que aún no tienen respuesta: 1) ¿Cuál de las ecuaciones de predicción del $\mathrm{VO}_{2 \max }$ validadas debería ser aplicada? 2) ¿Cuál de las ecuaciones validadas es mas precisa para estimar el $\mathrm{VO}_{2 \max }$ en adultos jóvenes? 3) ¿Cuál de las ecuaciones de predicción del $\mathrm{VO}_{2 \max }$ es más sensible y específica para diagnosticar SM u otro problema de salud en adultos jóvenes? 4) ¿Los CR propuestos por el Fitnessgram ${ }^{\circledR}$ son adecuados para ser aplicados en adultos jóvenes? Estas preguntas y los resultados de este estudio ponen de manifiesto la necesidad de profundizar la investigación. $\mathrm{Si}$ el objetivo es determinar el $\mathrm{VO}_{2 \max }$ de un sujeto, el criterio de elección de la ecuación sería utilizar aquella que fue validada en una población con similares características (edad, sexo, composición corporal, nivel de actividad física) y que presente el menor EEE. En adultos jóvenes varones la ecuación de Flouris ${ }^{30}$ es la que mostró el menor EEE en predecir el $\mathrm{VO}_{2 \max }$. Sin embargo, no ha sido estudiada la precisión para predecir el $\mathrm{VO}_{2 \max }$ de las diferentes ecuaciones aplicadas en el presente estudio en una misma muestra. En la actualidad no es posible determinar cuál de las ecuaciones validadas en adultos jóvenes es más precisa para detectar SM u otro problema de salud porque aún no se ha estudiado la sensibilidad y la especificidad de las diferentes ecuaciones para detectar factores de riesgo o una enfermedad específica. El $\mathrm{VO}_{2 \max }$ umbral o el $\mathrm{CR}$ a utilizar en adultos jóvenes es posible que difiera entre las distintas ecuaciones, como lo mostró el estudio de Moreira en adolescentes $^{24}$. Creemos que la validación y confección de los CR para la capacidad aeróbica en niños, adolescentes y adultos debería realizarse a partir del empleo de un test de campo y no de laboratorio como el que propone Fitnessgram $^{\circledR 2}$.

Una de las limitaciones de esta investigación fue no haber empleado una evaluación objetiva de los niveles de actividad física. También la imposibilidad de evaluar la precisión de las distintas ecuaciones predictivas del $\mathrm{VO}_{2 \max }$ para detectar SM o problemas cardiovasculares. Esta se debió principalmente a que algunos de los factores de salud que definen el SM no fueron evaluados. En relación a los niveles de capacidad aeróbica encontrados, es importante mencionar que el índice de respuesta del estudio podría condicionar los resultados a la hora de generalizarlos, ya que el $37,9 \%$ de los estudiantes invitados rechazaron participar. Es probable que los sujetos que no participaron tuvieran un nivel de condición física mas bajo.

En conclusión, los datos del presente estudio son los primeros en demostrar que el tipo de ecuación predictiva del $\mathrm{VO}_{2 \max } \mathrm{y}$ los $\mathrm{CR}$ del Fitnessgram $^{\circledR}$, modifican las clasificaciones de adultos jóvenes con capacidad aeróbica saludable o de RCM. Además una mayor proporción de mujeres en relación a los hombres fueron clasificadas con un nivel de capacidad aeróbica no saludable independientemente de la ecuación de predicción del $\mathrm{VO}_{2 \max }$ aplicada o el CR referencia del Fitnessgram ${ }^{\mathbb{R}}$ utilizado. Se necesitan más investigaciones que profundicen el estudio de los factores que pueden 
provocar un error en las clasificaciones a partir de la evaluación de la capacidad aeróbica. Futuros estudios deberían centrarse en analizar la utilidad clínica y la validez del $\mathrm{CN}$ $20 \mathrm{~m}$ para diagnosticar síndrome metabólico $\mathrm{u}$ otros problemas de salud en adultos jóvenes.

\section{AGRADECIMIENTOS}

Agradecemos a Jonatan Ruiz la revisión del borrador del manuscrito y haber respondido a nuestras inquietudes. A Marisa Tumino por su asistencia en el tratamiento estadístico. A los estudiantes que voluntariamente participaron de este estudio.

\section{BIBLIOGRAFÍA}

1. Ruiz JR. Health-related fitness assessment in childhood and adolescence: a European approach based on the AVENA, EYHS and HELENA studies. J Public Health. 2006; 14: 269-77.

2. Welk GJ, Laurson KR, Eisenmann JC, Cureton KJ. Development of youth aerobic-capacity standards using receiver operating characteristic curves. Am J Prev Med. 2011;41(4 Suppl 2):S111-6.

3. Taylor HL, Buskirk E, Henschel A. Maximal oxygen intake as an objective measure of cardio-respiratory performance. J Appl Physiol. 1955; 8: 73-80.

4. McLaughlin JE, Howley ET, Bassett DR Jr, Thompson DL, Fitzhugh EC. Test of the classic model for predicting endurance running performance. Med Sci Sports Exerc. 2010; 42(5):991-7.

5. Bassett DR, Howley ET. Maximal oxygen uptake: Classical versus contemporary viewpoints. Med Sci Sports Exerc. 1997; 29: 591-603.

6. Coyle EF. Integration of the physiological factors determining endurance performance ability. Exerc Sport Sci Rev. 1995; 23:25-63.

7. Blair SN, Kohl HW, Paffenbarger RS Jr, Clarke DG, Cooper KH, Gibbods LW. Physical fitness and all-cause mortality: a prospective study of healthy men and women JAMA. 1989; 262:2395-401.

8. LaMonte MJ, Blair SN. Physical activity, cardiorespiratory fitness, and adiposity: contributions to disease risk. Curr Opin Clin Nutr Metab Care. 2006;9(5):540-6.
9. Swain DP, Franklin BA. Comparison of cardioprotective benefits of vigorous versus moderate intensity aerobic exercise. Am J Cardiol. 2006;97(1):141-7.

10. Ortega FB, Ruiz JR, Castillo MJ, Sjöström M. Physical fitness in childhood and adolescence: a powerful marker of health. Int J Obes (Lond). 2008;32(1):1-11.

11. Secchi JD, García GC. Aptitud Física en Estudiantes de Educación Física, Medicina y Contador Público de la Universidad Adventista del Plata. G-SE. 20/03/2012. Disponible en: www.g-se.com/a/1395.

12. Garber CE, Blissmer B, Deschenes MR, Franklin BA, Lamonte MJ, Lee IM, et al. American College of Sports Medicine position stand. Quantity and quality of exercise for developing and maintaining cardiorespiratory, musculoskeletal, and neuromotor fitness in apparently healthy adults: guidance for prescribing exercise. Med Sci Sports Exerc. 2011;43(7):1334-59.

13. Carnethon MR, Gidding SS, Nehgme R, Sidney S, Jacobs DR Jr, Liu K. Cardiorespiratory fitness in young adulthood and the development of cardiovascular disease risk factors. JAMA. 2003;290(23):3092-100.

14. Sui X, LaMonte MJ, Laditka JN, Hardin JW, Chase $\mathrm{N}$, Hooker SP, et al. Cardiorespiratory fitness and adiposity as mortality predictors in older adults. JAMA 2007;298(21):2507-16.

15. Lee DC, Artero EG, Sui X, Blair SN. Mortality trends in the general population: the importance of cardiorespiratory fitness. J Psychopharmacol. 2010;24(4 Suppl):27-35.

16. Sloan RA, Sawada SS, Martin CK, Church T, Blair $\mathrm{SN}$. Associations between cardiorespiratory fitness and health-related quality of life. Health Qual Life Outcomes. 2009;7:47.

17. Sui X, Laditka JN, Church TS, Hardin JW, Chase N, Davis K, et al. Prospective study of cardiorespiratory fitness and depressive symptoms in women and men. J Psychiatr Res. 2009;43(5):546-52.

18. Berthoin S, Gerbeaux M, Turpin E. Comparison of two field tests to estimate maximum aerobic speed. J Sports Sci.1994;12:355-62.

19. García GC, Secchi JD. Relación de las velocidades finales alcanzadas entre el Course Navette de 20 metros y el test de VAM-EVAL. Una propuesta para predecir la velocidad aeróbica máxima. Apunts Med Esport. 2012. (in press) doi:10.1016/j.apunts.2011.11.004. 
20. Penry, JT, Wilcox AR, and Yun J. "Validity and reliability analysis of Cooper's 12-minute run and the multistage shuttle run in healthy adults." J Strength Cond Res. 2011;25(3): 597-605.

21. Morrow JR Jr, Zhu W, Franks BD, Meredith MD, Spain C.1958-2008: 50 years of youth fitness tests in the United States.Res Q Exerc Sport. 2009;80(1):1-11.

22. Zhu W, Mahar MT, Welk GJ, Going SB, Cureton $\mathrm{KJ}$. Approaches for development of criterion-referenced standards in health-related youth fitness tests. Am J Prev Med. 2011;41(4 Suppl 2):S68-76.

23. Lobelo F, Pate RR, Dowda M, Liese AD, Ruiz JR. Validity of cardiorespiratory fitness criterion-referenced standards for adolescents. Med Sci Sports Exerc. 2009;41(6):1222-9.

24. Moreira C, Santos R, Ruiz JR, Vale S, SoaresMiranda L, Marques AI, et al. Comparison of different $\mathrm{VO}_{2 \max }$ equations in the ability to discriminate the metabolic risk in Portuguese adolescents. J Sci Med Sport. 2011;14(1):79-84.

25. Welk GJ, De Saint-Maurice Maduro PF, Laurson KR, Brown DD. Field evaluation of the new FITNESSGRAM ${ }^{\circledR}$ criterion-referenced standards. Am J Prev Med. 2011;41(4 Suppl 2):S131-42.

26. Cureton KJ, Warren GL. Criterion-referenced standards for youth health-related fitness tests: a tutorial. Res Q Exerc Sport. 1990;61:7-19.

27. Léger L, Gadoury C. Validity of the $20 \mathrm{~m}$ shuttle run test with 1 min stages to predict $\mathrm{VO} 2 \mathrm{max}$ in adults. Can J Sport Sci.1989; 14(1):21-6.

28. Stickland MK, Petersen SR, Bouffard M. Prediction of maximal aerobic power from the $20-\mathrm{m}$ multistage shuttle run test.Can J Appl Physiol. $2003 ; 28(2): 272-82$.

29. Matsuzaka A, Takahashi Y, Yamazoe M, Kumakura N, Ikeda A, Wilk B, et al. Validity of the Multistage 20M Shuttle-Run Test for Japanese Children, Adolescents, and Adults. Pediatric Exercise Science, 2004;16:113-125.

30. Flouris AD, Metsios GS, Koutedakis Y. Enhancing the efficacy of the $20 \mathrm{~m}$ multistage shuttle run test.Br J Sports Med. 2005;39(3):166-70.

31. Ruiz JR, Ramirez-Lechuga J, Ortega FB, CastroPiñero J, Benitez JM, Arauzo-Azofra A, et al. Artificial neural network-based equation for estimating $\mathrm{VO}_{2}$ from the $20 \mathrm{~m}$ shuttle run test in adolescents. Artif Intell Med. 2008;44(3):233-45.
32. Chatterjee P; Banerjee AK.; Das P; Debnath, PA. Regression equation for the estimation of $\mathrm{VO}_{2 \max }$ in nepalese male adults. J. Hum. Sport Exerc. 2010;2:127-133.

33. Chatterjee P, Banerjee AK, Das P, Debnath PA. Regression equation for the estimation of maximum oxygen uptake in nepalese adult females. Asian J Sports Med. 2010;1(1):41-5.

34. Craig CL, Marshall AL, Sjöström M, Bauman AE, Booth ML, Ainsworth BE et al. International physical activity questionnaire: 12 -country reliability and validity. Med Sci Sports Exerc. 2003;35(8):1381-95.

35. Guidelines for Data Processing and Analysis of the International Physical Activity Questionnaire (IPAQ) Noviembre 2005. Disponible en: http://www.ipaq.ki.se/scoring.pdf

36. Heyward VH (2008). Evaluación de la aptitud física y prescripción del ejercicio. Madrid: Editorial Médica Panamericana. p.23-30.

37. ISAK International Standards for Anthropometric Assessment. International society for the advancement of kinanthropometry (ISAK). Adelaide: International Society for the Advancement of Kinathropometry; 2001.

38. Grant S, Corbett K, Amjadt AM, Wilson J and Aitchisont T. A comparison of methods of predicting maximum oxygen uptake. Br J Sports Med. 1995; 29: 147-152.

39. Kim J, Jung SH, Cho HC. Validity and reliability of shuttle-run test in Korean adults. Int J Sports Med. 2011;32(8):580-5

40. Gormley SE, Swain DP, High R, Spina RJ, Dowling EA, Kotipalli US, et al. Effect of intensity of aerobic training on $\mathrm{VO}_{2 \max }$. Med Sci Sports Exerc. 2008;40(7):133643

41. Swain DP. Moderate or vigorous intensity exercise: which is better for improving aerobic fitness? Prev Cardiol. $2005 ; 8(1): 55-8$.

42. Swain DP, Franklin BA. Comparison of cardioprotective benefits of vigorous versus moderate intensity aerobic exercise. Am J Cardiol. 2006;97(1):141-7.

43. Charkoudian N, Joyner MJ. Physiologic considerations for exercise performance in women. Clin Chest Med. 2004;25(2):247-55. 
Anexo 1

Ecuaciones predictivas del $\mathrm{VO}_{2 \max }$ para el Course Navette

\begin{tabular}{|c|c|c|c|c|}
\hline Estudio & Muestra & $\begin{array}{l}\text { Variables } \\
\text { ingresadas }\end{array}$ & \#Ecuación predictiva del $\mathrm{VO}_{2 \max \text { o pico }}$ & $\begin{array}{c}\mathrm{EEE}^{*} \\
\left(\mathrm{ml} / \mathrm{kg}^{-1} / \mathrm{min}^{-1}\right)\end{array}$ \\
\hline $\begin{array}{c}\text { Leger y Gadoury } \\
1989^{27}\end{array}$ & $\begin{array}{c}53 \text { y y } 24 \\
19-47 \text { años }\end{array}$ & Velocidad & $\begin{array}{l}\text { Hombres y mujeres: } \\
\mathrm{VO} 2 \mathrm{max}=6 \times \mathrm{V}-27,4\end{array}$ & 4,7 \\
\hline $\begin{array}{l}\text { Stickland et al. } \\
2003^{28}\end{array}$ & 60 y 62 & $\begin{array}{l}\text { velocidad, } \\
\text { número de } \\
\text { pasadas }\end{array}$ & $\begin{array}{l}\text { T: } \mathrm{VO} 2 \max =2,75 \times \mathrm{X}+28,8 \\
\text { ㅇ: } \mathrm{VO} 2 \max =2,85 \times \mathrm{X}+25,1\end{array}$ & $\begin{array}{l}\hat{\jmath}=4,07 \\
\phi=3,64\end{array}$ \\
\hline $\begin{array}{l}\text { Matsuzaka et al. } \\
2004^{29}\end{array}$ & $\begin{array}{l}56 \delta \text { y } 99 q \\
18-23 \text { años }\end{array}$ & $\begin{array}{c}\text { Sexo, } \\
\text { edad, } \\
\text { IMC, } \\
\text { velocidad, } \\
\text { número de } \\
\text { pasadas }\end{array}$ & $\begin{array}{c}\text { Hombres y mujeres: } \\
\text { a.VO2pico }=-2,19-3,46 \times \mathrm{S}-0,416 \times \mathrm{IMC}+5,22 \times \mathrm{V} \\
\text { b.VO2pico }=42,4-2,85 \times \mathrm{S}-0,488 \times \mathrm{IMC}+0,247 \times \mathrm{N}^{\circ} \\
\text { vueltas }\end{array}$ & $\begin{array}{l}\text { a. }=3,0 \\
\text { b. }=3,0\end{array}$ \\
\hline $\begin{array}{l}\text { Flouris et al. } \\
2005^{30}\end{array}$ & $\begin{array}{c}40 \AA \\
21,6 \pm 2,5 \text { años }\end{array}$ & Velocidad & $\delta: \mathrm{VO} 2 \mathrm{max}=\mathrm{V} \times 6,65-35,8$ & 1,7 \\
\hline Ruiz et al. $2008^{31}$ & $\begin{array}{c}122 \text { ○े y } 70 \text { q } \\
13-19 \text { años }\end{array}$ & & disponible en: http://www.helenastudy.com/scientific.php & 2,84 \\
\hline $\begin{array}{l}\text { Chatterjee et al. } \\
\qquad 2010^{32}\end{array}$ & $\begin{array}{c}40 \AA \\
20,4-24,9 \text { años }\end{array} \mid$ & Velocidad & $\mathrm{J}: \mathrm{VO} 2 \mathrm{max}=-16.961+5.068 \times \mathrm{X}$ & - \\
\hline $\begin{array}{l}\text { Chatterjee et al. } \\
\qquad 2010^{33}\end{array}$ & $\begin{array}{c}40 \stackrel{+}{ } \\
20,4-24,8 \text { años }\end{array}$ & Velocidad & $q: \mathrm{VO} 2 \max =-15.207+4.806 \times \mathrm{X}$ & - \\
\hline
\end{tabular}

*EEE = Error estándar de estimación. $\widehat{\delta}=$ hombres; $q=$ mujeres. \#Las siglas de las ecuaciones son las siguientes: Leger $^{27}$ : Donde $\mathrm{V}=$ velocidad de la última etapa completa en $\mathrm{km} \cdot \mathrm{h}-1$. Stickland ${ }^{28}$ : Donde $\mathrm{X}=$ corresponde a la última etapa completa, considerando hasta la mitad de la etapa alcanzada. Matsuzaka ${ }^{29}$ : Donde $S=\operatorname{sexo}(\hat{\delta}=0$ y $q=1)$; IMC $=$ índice de masa corporal; $\mathrm{V}=$ velocidad de carrera en $\mathrm{km} / \mathrm{h}^{-1} ; \mathrm{N}^{\circ}$ vueltas= número total de pasadas de 20 metros. Flouris $^{30}$ : Donde $\mathrm{V}=$ velocidad de la última etapa completa en $\mathrm{km} / \mathrm{h}^{-1}$. Chatterjee ${ }^{32,33}$ : Donde $\mathrm{X}=$ velocidad máxima que se alcanzó en el CN-20m en $\mathrm{km} / \mathrm{h}^{-1}$. 